\title{
The Evolution of Research on Abundances of Solar Energetic Particles
}

\author{
Donald V. Reames
}

Citation: Reames, D.V. The

Evolution of Research on Abundances of Solar Energetic Particles. Universe 2021, 7, 292. https://doi.org/10.3390/ universe7080292

Academic Editor: Ruiguang Wang

Received: 11 July 2021

Accepted: 5 August 2021

Published: 8 August 2021

Publisher's Note: MDPI stays neutral with regard to jurisdictional claims in published maps and institutional affiliations.

Copyright: (C) 2021 by the author. Licensee MDPI, Basel, Switzerland. This article is an open access article distributed under the terms and conditions of the Creative Commons Attribution (CC BY) license (https:// creativecommons.org/licenses/by/ $4.0 /)$.
IPST, University of Maryland, College Park, MD 20742, USA; dvreames@umd.edu

\begin{abstract}
Sixty years of study of energetic particle abundances have made a major contribution to our understanding of the physics of solar energetic particles (SEPs) or solar cosmic rays. An early surprise was the observation in small SEP events of huge enhancements in the isotope ${ }^{3} \mathrm{He}$ from resonant wave-particle interactions, and the subsequent observation of accompanying enhancements of heavy ions, later found to increase 1000 -fold as a steep power of the mass-to-charge ratio $A / Q$, right across the elements from $\mathrm{H}$ to $\mathrm{Pb}$. These "impulsive" SEP events have been related to magnetic reconnection on open field lines in solar jets; similar processes occur on closed loops in flares, but those SEPs are trapped and dissipate their energy in heat and light. After early controversy, it was established that particles in the large "gradual" SEP events are accelerated at shock waves driven by wide, fast coronal mass ejections (CMEs) that expand broadly. On average, gradual SEP events give us a measure of element abundances in the solar corona, which differ from those in the photosphere as a classic function of the first ionization potential (FIP) of the elements, distinguishing ions and neutrals. Departures from the average in gradual SEPs are also power laws in $A / Q$, and fits of this dependence can determine $Q$ values and thus estimate source plasma temperatures. Complications arise when shock waves reaccelerate residual ions from the impulsive events, but excess protons and the extent of abundance variations help to resolve these processes. Yet, specific questions about SEP abundances remain.
\end{abstract}

Keywords: solar cosmic rays; solar energetic particles; shock waves; coronal mass ejections; solar jets; solar flares; solar system abundances; solar wind

\section{Introduction}

Differential rotation of the ionized plasma in the Sun generates the solar magnetic field, and solar activity is driven by energy released in sudden changes and reconnections of that field. This energy release appears in the form of solar flares and jets, and in the detachment of coronal mass ejections (CMEs) that expand into interplanetary space. Solar cosmic rays or solar energetic particles (SEPs), as they are commonly known, can be associated with all of these processes, from the direct reconnection and wave-particle resonances in flares and jets to the shock waves driven by fast CMEs. The largest events contain of a burst of SEPs up to $\mathrm{GeV}$ energies lasting several days and spanning nearly $360^{\circ}$ in solar longitude. SEPs threaten Mars-bound astronauts with hazardous radiation.

The physical processes that accelerate SEPs are of general astrophysical interest and, at a minimum, they apply to most stars in the universe. While we can see evidence for some of these processes elsewhere, only the Sun also provides us with a direct physical sample of the energetic particles themselves, allowing the observation of their spectra and abundances in addition to the radio, optical, $\mathrm{X}$-ray, and $\gamma$-ray photons they emit, thus providing a multi-messenger view of the physics. In recent years, as the study of SEPs has advanced, we have found that the particles themselves carry information on conditions in their coronal source in addition to the physical processes of their acceleration.

The purpose of this article is to acknowledge sixty years of progress in this field, to review the controversies and accomplishments, and to highlight remaining questions. 


\section{The First SEPs}

SEPs were first observed by Scott Forbush [1] in Cheltenham, Maryland using groundbased ion chambers which measured ionization of the cascade of secondary particles produced when $\mathrm{GeV}$ protons strike the upper atmosphere. This system measured ionization, proportional to the number of arriving galactic cosmic rays (GCRs), and its decrease when GCRs were screened out by magnetic fields in the structures we now call coronal mass ejections (CMEs). Forbush observed sharp increases in particle intensity from SEPs, accompanied by solar flares, which preceded these "Forbush decreases" in February and March 1942, and again in July 1946. These SEP increases were also seen simultaneously in Greenland and New Zealand. Thus, the study of SEPs began with these ground-level events (GLEs), the largest, most energetic SEP events we see. It would be many years before Kahler et al. [2] began to firmly establish that the SEPs in these large "gradual" or long-duration events were actually accelerated, with a $96 \%$ association, at shock waves driven by wide, fast CMEs similar to those that produced the Forbush decreases. The obvious, bright flares that were observed by optical instruments were a consequence of regions of energy containment on closed loops in the huge magnetic reconnections involved.

The first measurement of the abundances of chemical elements in SEP events was made by Fichtel and Guss [3] using nuclear emulsions on a 4 min sounding rocket flight from Ft. Churchill, Manitoba into the SEP event of 3 September 1960. They observed C, N, $\mathrm{O}, \mathrm{Ne}, \mathrm{Mg}, \mathrm{Si}$, and $\mathrm{S}$, beginning a sixty-year effort to compare these SEP abundances with those of the solar photosphere and corona, where element abundance measurements were also slowly improving.

The quality and quantity of measurements improved greatly once Si solid-state telescopes were flown regularly on satellites. A review of these measurements by Meyer [4] and observations by Breneman and Stone [5] established that the relative abundances of elements in these large gradual events consist of two factors: (1) average abundances that differed from solar photospheric abundances as a function of the first ionization potential (FIP) of the elements, and (2) additional element enhancements that depend upon a power law in the mass-to-change ratio, $A / Q$. For element abundances compared at a single velocity, $A / Q$ represents the effects that depend upon an ion's magnetic rigidity. This second factor could vary from event to event and even with time during events. A modern version of the "FIP effect" is shown in Figure 1 and represents the abundances in the SEP-determined corona, relative to the two measurements of the solar photosphere. SEP abundances in Figure 1 [6-8] provide the reference for all subsequent discussions below of abundance enhancements or suppressions.

In the current theory by Laming, the low-FIP $(<10 \mathrm{eV})$ elements which are ionized in the photosphere are subject to ponderomotive force of Alfvén waves, while the neutral highFIP elements are not [9]. The forces differ for closed magnetic loops, near active regions, where the Alfvén waves can resonate with the loop length. Differences in the FIP pattern between SEPs and the solar wind are seen $[10,11]$, and have been explained theoretically by closed and open field lines [9,12]. The two different photospheric abundance sets depend upon the choice of spectral lines used for the measurement. 


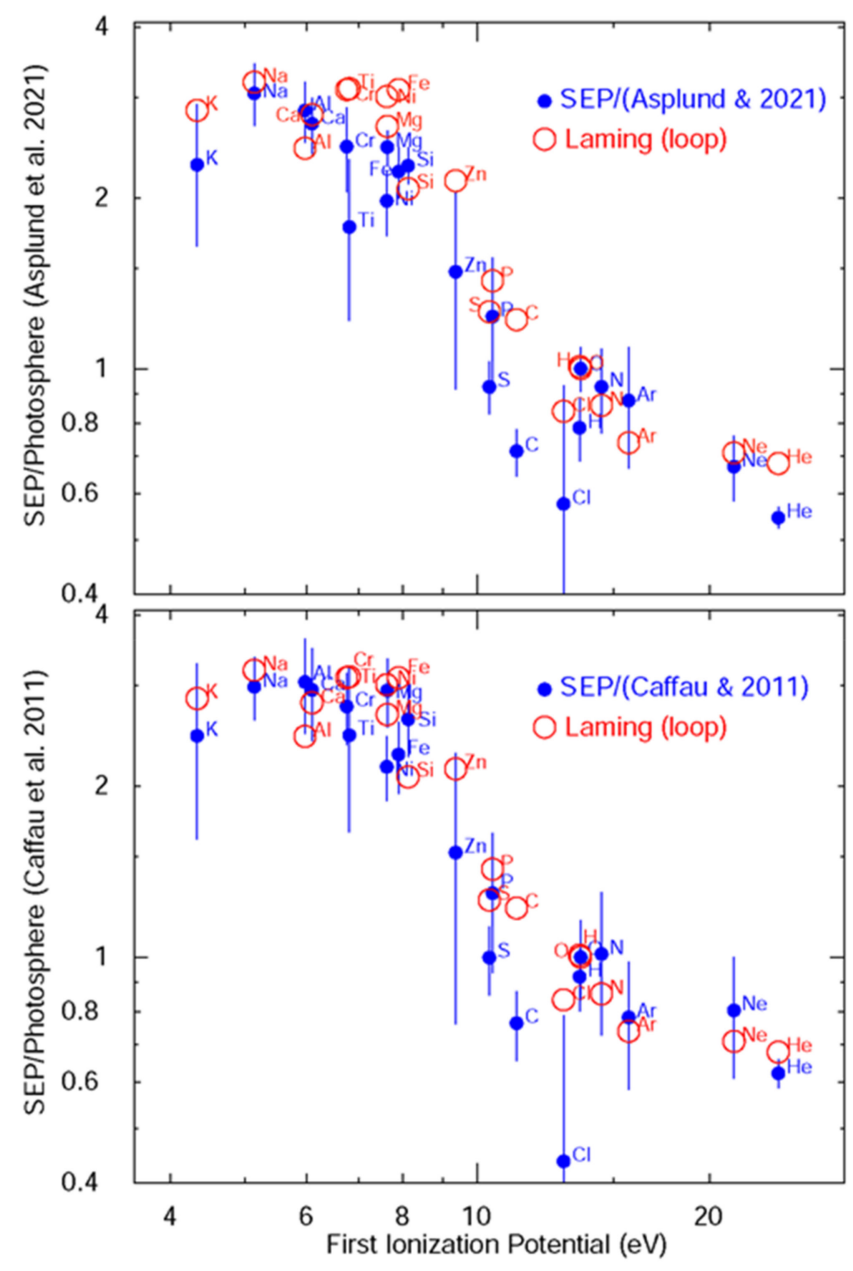

Figure 1. Average SEP abundances [6-8], relative to O, divided by the corresponding solar photospheric abundances $[13,14]$ are plotted vs. FIP (blue filled circles) and compared with theoretical calculations [12] for closed loops (open red circles). Points are labeled by element names.

\section{Electrons}

An important source of early information on particles from the Sun was obtained from radio observations. As electrons of 10-100 keV stream out from the Sun along the interplanetary field lines, they excite waves at the local plasma frequency, which decreases as the square root of the local plasma density. Thus, electrons streaming from sources near the Sun produce radio type III bursts, which drift rapidly to a lower frequency as the electrons traverse rapidly decreasing plasma density, while similar electrons at coronal or interplanetary shock waves produce type II bursts, which move out more slowly at the speed of the shock. The classic review by Wild, Smerd, and Weiss [15] initiated the idea of two solar particle sources, associating type III bursts with sources of electrons near the Sun, and type II bursts with shock waves that accelerated primarily protons. Subsequent early observations of electrons in space seemed to confirm this idea [16]. The type III bursts were thought to involve "pure" electron events, without ions, but that was before the measurements of ${ }^{3} \mathrm{He}$.

\section{4. ${ }^{3}$ He-Rich Events}

Nearly every scientist observing SEPs in the early days had previous experience with GCRs. After acceleration from supernovae, GCRs spend $\sim 10^{7}$ years in space, where they fragment against interstellar $\mathrm{H}$ to produce significant abundances of secondary ${ }^{2} \mathrm{H}$ and ${ }^{3} \mathrm{He}$ ions as well as isotopes of Li, Be, and B. When Hsieh and Simpson [17] measured ${ }^{3} \mathrm{He}$ 
in the SEPs at a ratio of ${ }^{3} \mathrm{He} /{ }^{4} \mathrm{He}=(2.1 \pm 0.4) \times 10^{-2}$, the automatic reaction was that SEPs had fragmented during traversal of a small amount of matter.

Then, even higher abundances of ${ }^{3} \mathrm{He}$ began to appear. Serlemitsos and Balasubrahmanyan [18] found a SEP event with ${ }^{3} \mathrm{He} /{ }^{4} \mathrm{He}=1.52 \pm 0.10$ but with ${ }^{3} \mathrm{He} /{ }^{2} \mathrm{H}>300$. More ${ }^{3} \mathrm{He}$ than ${ }^{4} \mathrm{He}$ but no ${ }^{2} \mathrm{H}$ ? This was not fragmentation. Then, there were limits established on Be/O and B/O in SEP events that were found to be $<2 \times 10^{-4}[19,20]$. This was not fragmentation; this was a completely new resonance phenomenon drastically and selectively enhancing the ${ }^{3} \mathrm{He}$.

These ${ }^{3} \mathrm{He}$-rich events would soon be shown to have increased abundances of heavy elements as well, such as a $\sim 10$-fold increase in Fe/O [21]. This was soon to be perceived as a power law increase in $A / Q$ [22] and, when measurements of heavy elements became available [23-26], would rise as the $3.64 \pm 0.15$ power [26] across the periodic table to become a factor of $\sim 900$ increase in $(76 \leq Z \leq 82) / O$, with the $Q$-values of the elements being determined at a temperature of $\sim 3 \mathrm{MK}$, where the elements $\mathrm{He}, \mathrm{C}$ and even $\mathrm{O}$ are fully ionized. In the solar corona, the pattern of ionization states determines the temperature, and conversely, the abundances based upon power laws in $A / Q$ require changes in $A / Q$ for relative enhancements.

A sample of ${ }^{3} \mathrm{He}$-rich events of increasing intensity is shown in Figure $2 .{ }^{3} \mathrm{He}$ equals or exceeds ${ }^{4} \mathrm{He}$ in the first three events, but is smaller (or unmeasurable) in the larger events. ${ }^{3} \mathrm{He}$ is found to be limited, as if all the ${ }^{3} \mathrm{He}$ in the acceleration volume is depleted in large events $[27,28]$. Except in the smallest event where it is unmeasured, we find that $\mathrm{Fe} / \mathrm{O} \approx 1$, vs. $0.131 \pm 0.006$ in the reference gradual SEP abundances. When the intensities become large enough, heavy elements begin to appear. See the review by Mason [29] for more information.

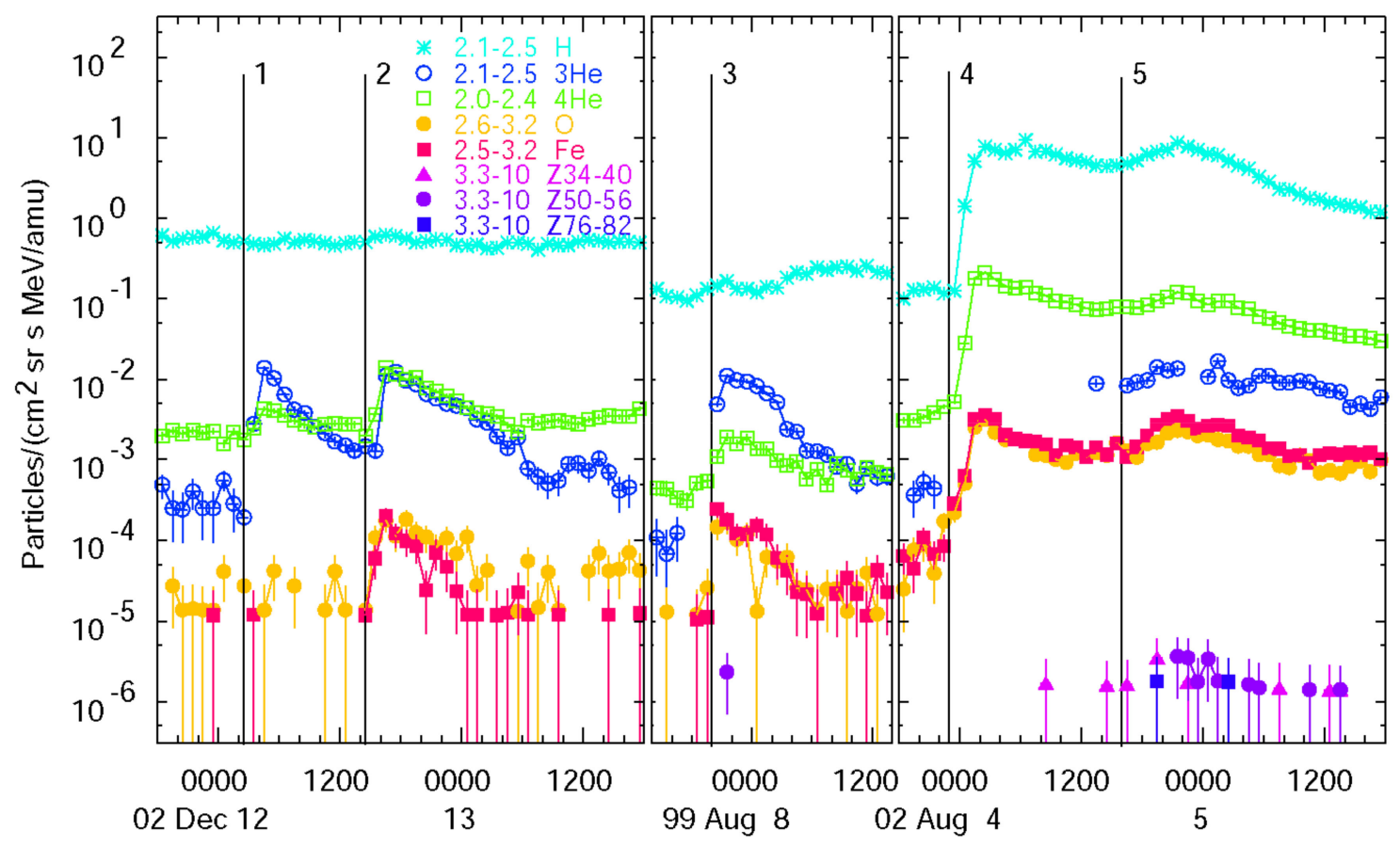

Figure 2. Element intensities at the indicated $\mathrm{MeV} \mathrm{amu}^{-1}$ are shown in a sample of ${ }^{3} \mathrm{He}$-rich or "impulsive" SEP events of increasing intensity. ${ }^{3} \mathrm{He}$ exceeds ${ }^{4} \mathrm{He}$ in Events 1 and 3 . Fe and $\mathrm{O}$ are below the background level in Event 1 , but Fe/O $\approx 1$ in the remaining events. $\mathrm{H}$ is below the background level in Events $1-3 .{ }^{3} \mathrm{He} /{ }^{4} \mathrm{He}<0.1$ in Event 4 and is not plotted because it falls below the possible resolution error; it is $\approx 0.1$ in Event 5 and high- $Z$ heavy elements are beginning to appear [24]. Other properties of the last four of these events are listed [26].

As measurements improved, the ${ }^{3} \mathrm{He}$-rich events were associated with the $10-100 \mathrm{keV}$ electron events [30] that produce the radio type III bursts [31], once thought to be "pure" 
electron events. Many of the theories to explain ${ }^{3} \mathrm{He}$ enhancements invoked preheating of the ions by selective absorption of various wave modes at the ${ }^{3} \mathrm{He}$ gyrofrequency [8,32-39] and acceleration by an unspecified mechanism. However, Temerin and Roth [40] suggested full acceleration of the ${ }^{3} \mathrm{He}$ by resonant absorption of electromagnetic ion cyclotron (EMIC) waves produced by the observed electron beams, while the ions were mirrored in the magnetic field.

However, all of the resonance theories attempting to explain the sharp increase in ${ }^{3} \mathrm{He}$ failed to explain the smoothly increasing enhancements of heavy elements. These enhancements were explained by simulations that showed the scattering of ions back and forth in the collapsing islands of magnetic reconnection [41], which predicted the power law dependence on $A / Q$. Similar considerations even apply to electron acceleration [42], but reconnection calculations have not yet explained ${ }^{3} \mathrm{He}$.

Measurements of the SEP ionization states of the elements up to Fe were possible below $\sim 2 \mathrm{MeV} \mathrm{amu}^{-1}$, and early measurements $[43,44]$ showed average values of $Q_{\mathrm{Si}}=11.0 \pm 0.3$ and $Q_{\mathrm{Fe}}=14.1 \pm 0.2$ for large gradual events to be obtained, suggesting a source temperatures of $\approx 2 \mathrm{MK}$. ${ }^{3} \mathrm{He}$-rich events, however, showed $Q_{\mathrm{Si}} \approx 14$ and $Q_{\mathrm{Fe}}=20.5 \pm 1.2$. Either, this source was very hot, $>10 \mathrm{MK}$, or the ions were stripped after acceleration. Unfortunately, the first interpretation, suggesting a hot flare origin, was generally accepted for many years; then, it was found by DiFabio et al. [45] that the ionization states of Fe increased with ion energy, as would be expected if the ions passed through enough material after acceleration to attain a velocity-dependent equilibrium charge state. This would occur if the ions had been accelerated at a height of $\sim 1.5$ solar radii in impulsive events. The amount of material required to strip $F e$ is too small to cause nuclear fragmentation or energy loss of high- $Z$ ions that would disrupt $A / Q$ power laws.

The result of DiFabio et al. [45] was important, because, otherwise, the highly ionized ions were not compatible with the abundances. If all the elements up to Si were fully ionized, with $A / Q=2$, how could $\mathrm{Ne}, \mathrm{Mg}$, and Si be enhanced by a factor of $\sim 2.5$ relative to $\mathrm{C}, \mathrm{N}$, and $\mathrm{O}$, while Fe was enhanced by a factor of $\sim 7$ [22]? This pattern was interpreted as suggesting that $\mathrm{He}, \mathrm{C}, \mathrm{N}$, and $\mathrm{O}$ were fully ionized, and $\mathrm{Ne}, \mathrm{Mg}$, and $\mathrm{Si}$ were in especially stable configurations with two orbital electrons. This pattern occurred in a temperature range of 3-5 MK [22]. It also meant that a best fit to the power-law of abundance enhancements vs. $A / Q$ could be used to determine a likely source temperature. Just try many temperatures and choose the best fit. This was a powerful new idea; abundances could determine a source temperature even after electrons are later stripped off the ions.

\section{The Solar-Flare Myth}

The small ${ }^{3} \mathrm{He}$-rich, Fe-rich "impulsive events" were clearly different from the large "proton events" or "gradual" events that lasted for days and spanned nearly $360^{\circ}$ in solar longitude [8]. This spread of particles was once explained by the "bird cage" model [46], where particles followed arcades of magnetic loops seen in active regions spreading like the wires of a bird cage, somehow allowing SEPs to hop from structure to structure at the footpoints of the loops and so on across the face of the Sun. Then, Kahler et al. [2] found a $96 \%$ correlation between large gradual SEP events and wide, fast CMEs. The shock waves driven by CMEs could easily cross field lines, accelerating particles as they went. Mason, Gloeckler, and Hovestadt [47] said the SEP abundance variations showed none of the magnetic rigidity dependence that a bird cage model required; they supported large-scale shock acceleration. The distribution of abundances of Fe/O showed a bipolar distribution [48]; the Fe-rich branch was ${ }^{3} \mathrm{He}$-rich, electron-rich, and proton-poor in comparison with the Fe-poor branch. Gradual events were associated with shock waves, while impulsive events were associated first with flares, and much later, with solar jets, as we will see below [49].

By the 1990s, the bird cage model seemed to have lost credence and impulsive and gradual SEP events were often distinguished in the literature, but Gosling's review article entitled "The Solar Flare Myth" [50,51], describing the ascendance of CMEs, caused widespread controversy. For example, it was found to "wage an assault on the last 30 years 
of solar-flare research" [52]. If flares did not accelerate astronaut-threatening radiation, perhaps flare research would not even be funded! In a subsequent discussion, commissioned by Eos, Hudson [53] argued that the term "flare" should be broadened to include CMEs and related shocks and SEPs; Miller [54] argued that, since flares were more numerous, they were better subjects for acceleration studies; and Reames $[55,56]$ argued that all SEP sources were interesting, but for SEPs, it was very important to know the spatial extent of a source. Subsequently, the importance of CME-driven shock waves received wider recognition.

Nevertheless, the distinction of sources using abundances was not complete. Shock waves in gradual SEP events were quite able to reaccelerate residual suprathermal ions left over from previous impulsive SEP events [57], sometimes even preferentially, as when the fast pre-accelerated impulsive ions were better able to overtake the shock from downstream, e.g., when the magnetic field was quasi-perpendicular to the shock normal [58-60]. It became necessary to discuss the seed population of ions available for shock acceleration, residual impulsive suprathermal ions in addition to ambient coronal plasma. In addition, pools of impulsive suprathermal ions seemed to collect quite often from many small impulsive events that were much more numerous than the larger events we could resolve [61-66].

\section{Jets}

Thus, it once seemed possible to distinguish impulsive and gradual SEP events by abundances. An event with enhanced ${ }^{3} \mathrm{He}$ and Fe was impulsive, was it not? However, as we have just seen, it is not so simple, since shocks can accelerate residual suprathermal ions, sometimes preferentially.

In the next shock to simplicity, we found that impulsive events can also have associated CMEs, even fast CMEs that drive shocks [67]. These seemed to be narrow CMEs associated with solar jets. Separately, the non-relativistic electron events that produce type III radio bursts had also been associated with solar jets [68]. Electrons and type III bursts had long been associated with impulsive SEP events [30,31]. Jets involve magnetic reconnection on open field lines, so the SEPs easily escape without significant heating. In contrast, flares actually require the plasma and SEPs to be trapped on closed loops where they produce the heating and X-ray Bremstrahlung that drive the sudden burst of photons where only neutrals can escape. Measurements of $\gamma$-ray lines in large flares [69] have shown that the accelerated "beam" is both ${ }^{3} \mathrm{He}$-rich $[70,71]$ and Fe-rich [72], similarly to impulsive SEPs in space.

SEP events can now be traced to their sources [26,73-75] and impulsive SEP events are routinely associated with individual solar jets [76-78]. See the review by Bučík [79] for more information. Impulsive SEP source regions often have characteristics of both flares and jets when the reconnection involves both open and closed field regions, as jet models predict. In these cases, the open regions can be $\sim 2.5 \mathrm{MK}$ and emit in the EUV, while the nearby closing field regions trap SEPs, heating to $>10 \mathrm{MK}$ and emitting X-rays. As event size increases, both SEPs and X-rays increase, but they involve different spatial regions in the source.

The CMEs from jets that are fast enough to drive shock waves can reaccelerate the SEPs produced in the reconnection event, essentially maintaining much of the increase in heavy-ion abundance with $A / Q$ that is in the impulsive component, but these shock waves can also sample the ambient coronal plasma. It turns out that the largest impulsive events, usually with fast CMEs, have an extra component of protons, a "proton excess", as seen in the comparative samples in Figure 3.

Here, the power law fits of the event-averaged enhancements in abundance, relative to the SEP reference abundances (Figure 1), are fit vs. $A / Q$ of the elements $6 \leq Z \leq 56$. For each event, fits are obtained using $Q$ values at several temperatures, and the temperature providing the best fit is selected, although it has been shown that most impulsive events have a limited range of $T \approx 2.5-3.2 \mathrm{MK}[80,81]$. When this best-fit line is extended down to protons at $A / Q=1$, the fit at $\mathrm{H}$ is good in many small events; Reames [82] labeled these 
SEP1 events. Other, larger events show a large proton excess and are distinguished as SEP2 events. Samples of each are shown in Figure 3 with the associated CME speeds listed, if any.

Impulsive SEP Events
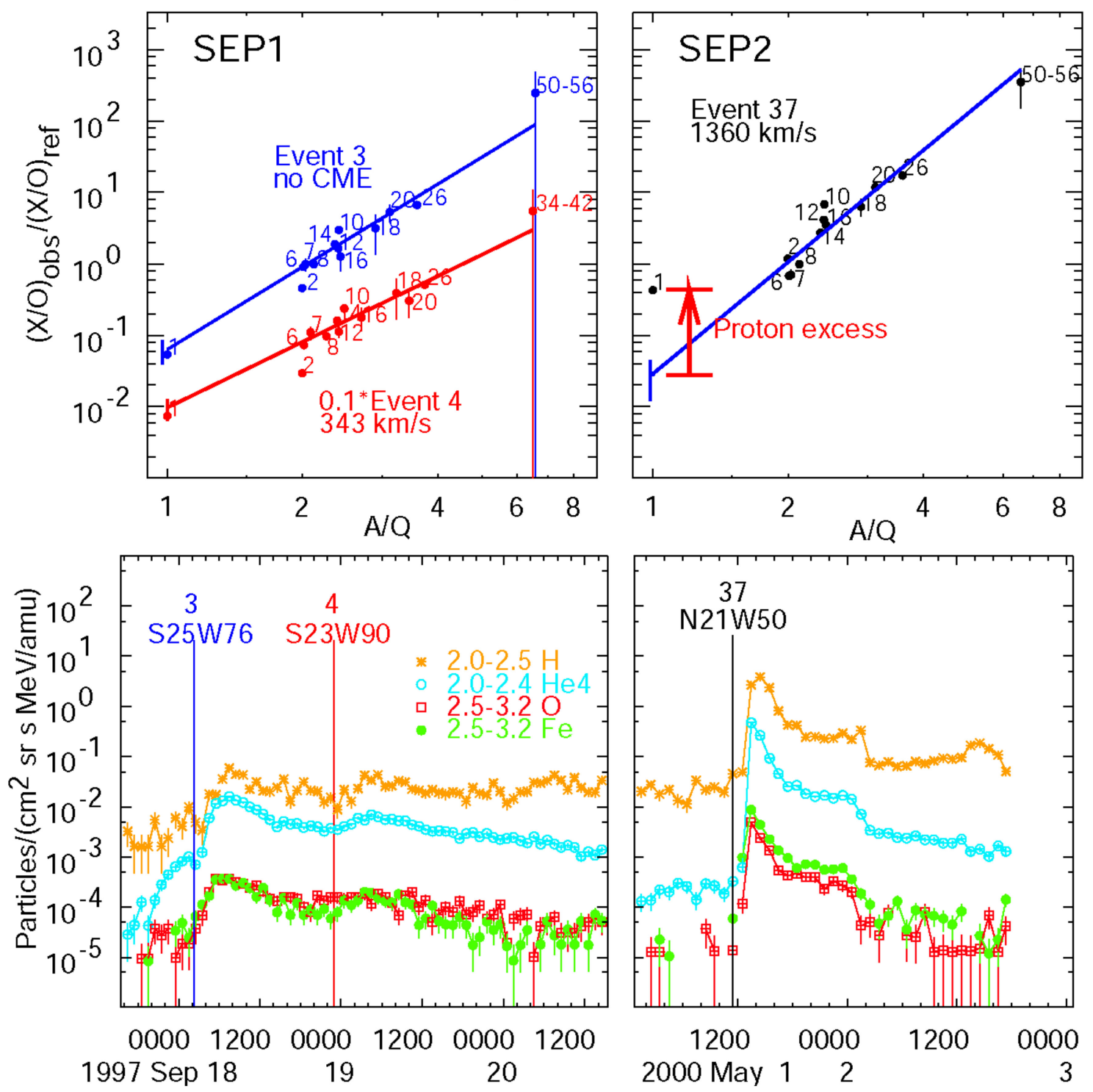

Figure 3. The lower panels show time histories of $\mathrm{H},{ }^{4} \mathrm{He}, \mathrm{O}$ and $\mathrm{Fe}$, at the indicated $\mathrm{MeV}_{\mathrm{amu}}{ }^{-1}$, for three impulsive SEP events, two small SEP1 events on the left and a larger SEP2 event on the right. Event numbers shown above source coordinates refer to the event list [26]. Power-law fits to the abundance enhancements, noted by $Z$, in each event, are shown in the upper panels and CME speeds are listed when CMEs are seen. The right-hand Event 37 is an SEP2 class event because of its large proton excess noted. For the SEP1 events on the left, the protons abundances lie on the extrapolated power-law fits $[82,83]$.

For a large sample of impulsive SEP events, Figure 4a shows how the distribution of event size and CME speed depends upon the measured proton excess. Figure $4 \mathrm{~b}$ shows a probable explanation; when a fast shock wave is present, protons from the ambient coronal plasma may dominate, while heavy ions are dominated by SEP1 ions pre-accelerated and pre-enhanced at the magnetic-reconnection site, i.e., a complex seed population. 

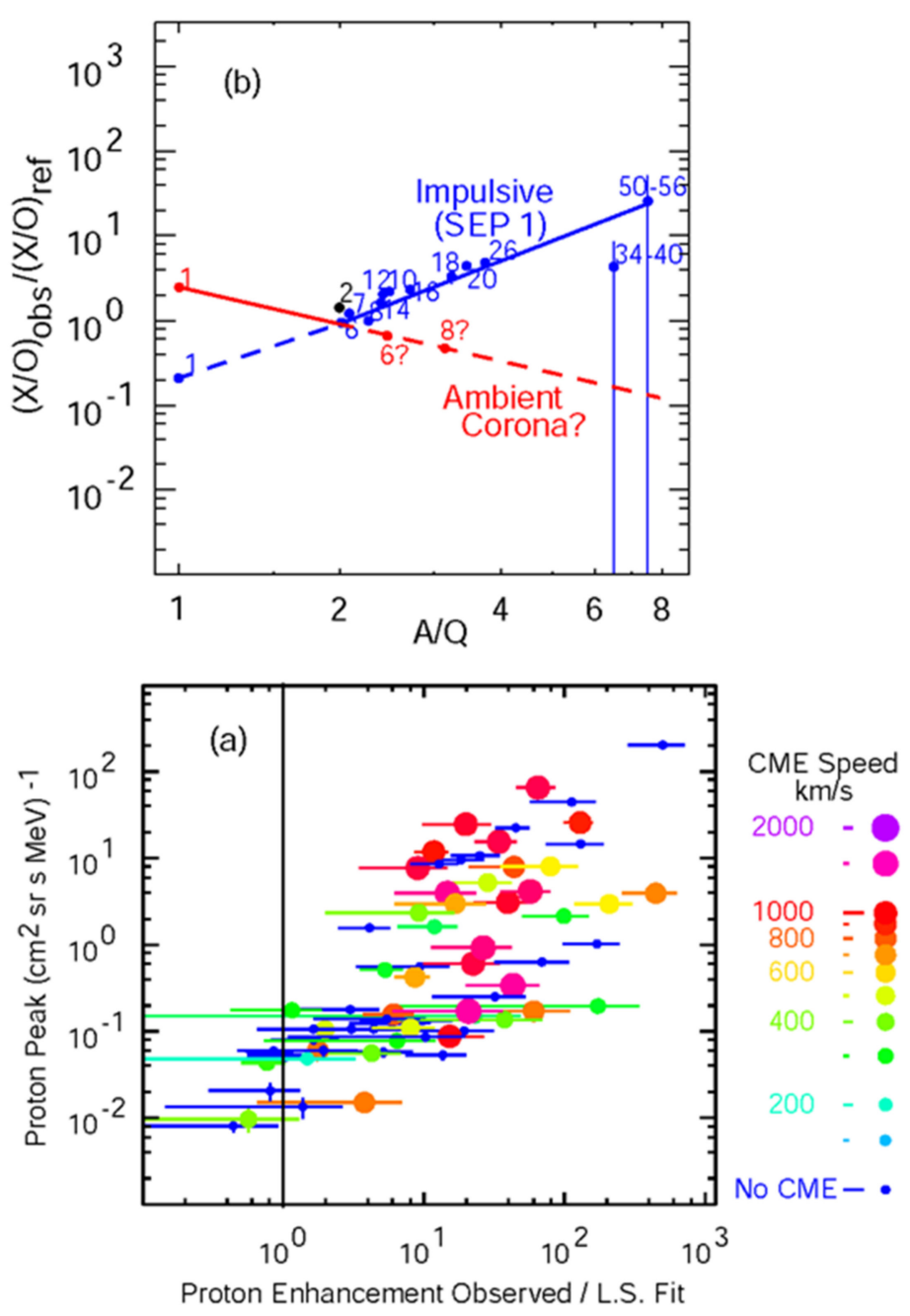

Figure 4. Panel (a) shows the peak proton intensity at $2-2.5 \mathrm{MeV}$ vs. the proton excess relative to the $Z>2$ power-law fit for 90 impulsive SEP events with the symbol size and color determined by CME speed as shown. Panel (b) suggests two possible contributions, impulsive SEP1 seed particles (blue) and ambient coronal seed particles (red), to the plot of enhancement vs. $A / Q$ for the shock-enhanced SEP2 impulsive events [82,83].

Recently, Bučík et al. [78] found source temperatures of 2-2.5 MK, using the differential emission measure technique on the extreme ultraviolet (EUV) images of coronal source regions of 24 solar jets associated with ${ }^{3} \mathrm{He}$-rich events. This agrees quite well with the dominant 2.5 and 3.2 MK source temperatures found from impulsive SEP abundances by Reames et al. [80,84], and tends to confirm the use of best-fit power laws of abundance enhancements vs. $A / Q$ to estimate source temperatures.

\section{Gradual SEP Events}

Shock acceleration in gradual SEP events has received considerable attention historically [85-92], and many reviews are available [8,27,93-95]. Many advances in our understanding come from features other than element abundances, including the spatial distributions of protons [96-100], the correlations of SEP and CME properties [101,102], onset timing [103-106], electron/proton ratios [107,108] or GLE events and spectral breaks [109-111]. Gradual events have also provided a basis for studies of the coronal FIP effect described above.

Departures from the coronal abundances behave as power laws in $A / Q$ in gradual SEP events, as was found in 1985 by Breneman and Stone [5]. In some events, pitch-angle scattering of particles during transport can change the powers to enhance heavy elements early in events and suppress them later. For example, if Fe scatters less than O, Fe/O may 
be enhanced early in an event, as Fe preferentially arrives early, but will be depressed later as more $\mathrm{O}$ arrives while $\mathrm{Fe}$ has been depleted. Solar rotation can spread this temporal behavior into a longitude dependence. Figure 5 shows an analysis of the gradual SEP event of 23 January 2012 [112].

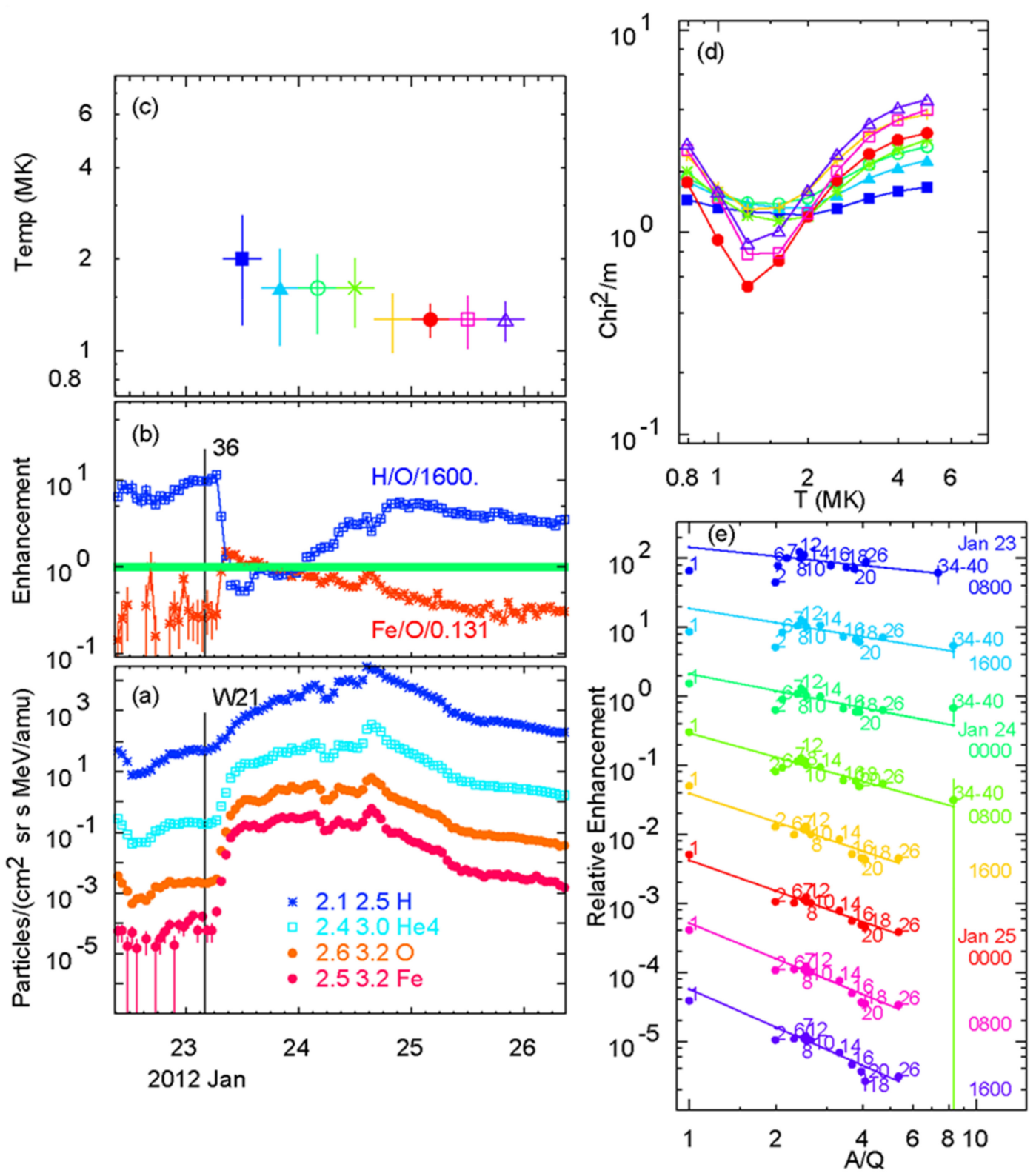

Figure 5. (a) Intensities of $\mathrm{H}, \mathrm{He}, \mathrm{O}$, and Fe vs. time. (b) Normalized abundance enhancements $\mathrm{H} / \mathrm{O}$ and $\mathrm{Fe} / \mathrm{O}$ vs. time. (c) Best-fit temperatures vs. time for the 23 January 2012 SEP event. (d) $\chi^{2} / m$ vs. $T$ for each $8 \mathrm{~h}$ interval. (e) Enhancements, labeled by $Z, v$ vs. $A / Q$ for each $8 \mathrm{~h}$ interval shifted $\times 0.1$, with best-fit power law for elements with $Z \geq 6$ extrapolated down to $\mathrm{H}$ at $A / Q=1$. Colors correspond for the eight intervals in (c-e) and symbols in (c,d); times are also listed in (e). Event onset is flagged with solar longitude in (a) and the event number from the list of 45 events [113] in (b) [112].

Figure $5 \mathrm{a}$ shows the general time behavior of the event, while Figure $5 \mathrm{~b}$ shows the variation of normalized $\mathrm{Fe} / \mathrm{O}$ and $\mathrm{H} / \mathrm{O}$ ratios. Figure $5 \mathrm{c}$ identifies the analyzed time intervals and shows the resulting best-fit temperatures for each. Figure $5 \mathrm{~d}$ shows how the quality of the least-squares fit to enhancement vs. $A / Q$ varies with temperature for each time interval. The temperatures in Figure $5 \mathrm{c}$ are chosen at the minima in Figure $5 \mathrm{~d}$. The best-fit power laws for each time interval are shown in Figure 5e, passing through element enhancements, labeled by $Z$. 
For the first few intervals in Figure 5e, the $A / Q$ dependences are very flat, so the assigned values of $A / Q$ for each ion make little difference, and hence the curves in Figure $5 \mathrm{~d}$ are very shallow and the temperatures are poorly determined and have large errors. Later on, the slopes steepen. When the power law dependences are steeper (either positive or negative), the $A / Q$-values matter, and temperatures are better determined. However, temperatures usually remain consistent during gradual events, even though the slopes of the power law fits often vary. Note in Figure 5e that the protons fit reasonably well on each of the fit lines determined from the elements with $6 \leq Z \leq 56$.

Reames [113] found that in a list of 45 gradual SEP events, $69 \%$ had $0.8 \leq T \leq 1.6 \mathrm{MK}$ and no significant proton excess, while $24 \%$ had $2 \leq T \leq 4$ MK with enhancements rising with $A / Q$, similarly to impulsive events, and also had substantial proton excesses. For these latter events, called SEP3 events [82], impulsive suprathermal seed ions, with enhancements rising with $A / Q$, dominated the enhancements for $Z>2$, while protons from the ambient coronal plasma dominated $H$. In these events, shocks of moderate strength apparently traversed pools of impulsive suprathermal ions. Overall, 69\% of the SEP4 events were accelerated by strong shocks traversing ambient coronal plasma and any impulsive suprathermals present might be included, but were overwhelmed.

The impulsive SEP2 events and the gradual SEP3 events seem similar in temperature range and proton excess. How can we distinguish them? The SEP2 events involve a single jet source with a fast CME-driven shock where there are local abundance variations, e.g., from the local plasma conditions, that are found to have $\sim 30 \%$ variations from event to event. In the SEP3 events, the fast shock waves traverse the accumulated pools of impulsive suprathermal ions where the abundances are averaged over many jets and variations are seen to be more like $\sim 10 \%$. If these pools average over $N$ jets, we might expect the variations be reduced by a factor of $\sqrt{ } N$, so we estimate $N \sim 10$. As stated previously, there is considerable observational evidence that ${ }^{3} \mathrm{He}$-rich, Fe-rich pools of suprathermal ions are common [61-66], suggesting that there are many small jets (nanojets?) occurring with high frequency, producing small SEP1 events that we are unable to resolve individually. Figure 6 compares the spread in normalized abundance ratios $\mathrm{O} / \mathrm{C}$ vs. $\mathrm{C} /{ }^{4} \mathrm{He}$ for these two sources that are relatively hot, where $\mathrm{He}, \mathrm{C}$, and $\mathrm{O}$ are most likely to be fully ionized. Impulsive events with large statistical errors have been omitted; the large spread of the remaining events is not due to their statistical errors.

Abundance patterns in SEP events can be affected by seed populations, by acceleration, and by scattering during transport [114]. For impulsive events, it has been found [115] that transport is nearly scatter free, i.e., the scattering mean free path is $>1 \mathrm{AU}$, in most events. In impulsive and small gradual events [114], the ions stream outward, as focusing on the divergent magnetic field overcomes scattering. In gradual events of moderate size, abundances tend to retain the character of the source, or of the seed population in SEP3 events, only weakly modified by transport, e.g., minimal changes of powerlaw slope with time [114]. However, in large SEP events, wave amplification by the streaming protons [116] becomes a significant factor. As proton intensities increase, wave growth increases until the scattering reduces the streaming to produce equilibrium at the "streaming limit" that modifies abundances, limits intensities, and flattens spectra early in large SEP4 events [117-125]. These events can causes dramatic changes in the power law slope, with time or spurious variations in the proton excess [114,125]. Since scattering depends upon past and present proton intensities, it can become a strong function of space, time, and particle rigidity [120-122]. The wave number of the resonant waves $k \approx B / \mu P$, where $B$ is the field strength, $P$ is the particle rigidity and $\mu$ is its pitch-angle cosine [119-121]. Other particle species or energies resonate with waves generated by protons at the same $k$. 
(b) SEP3 Gradual - wide, fast CME
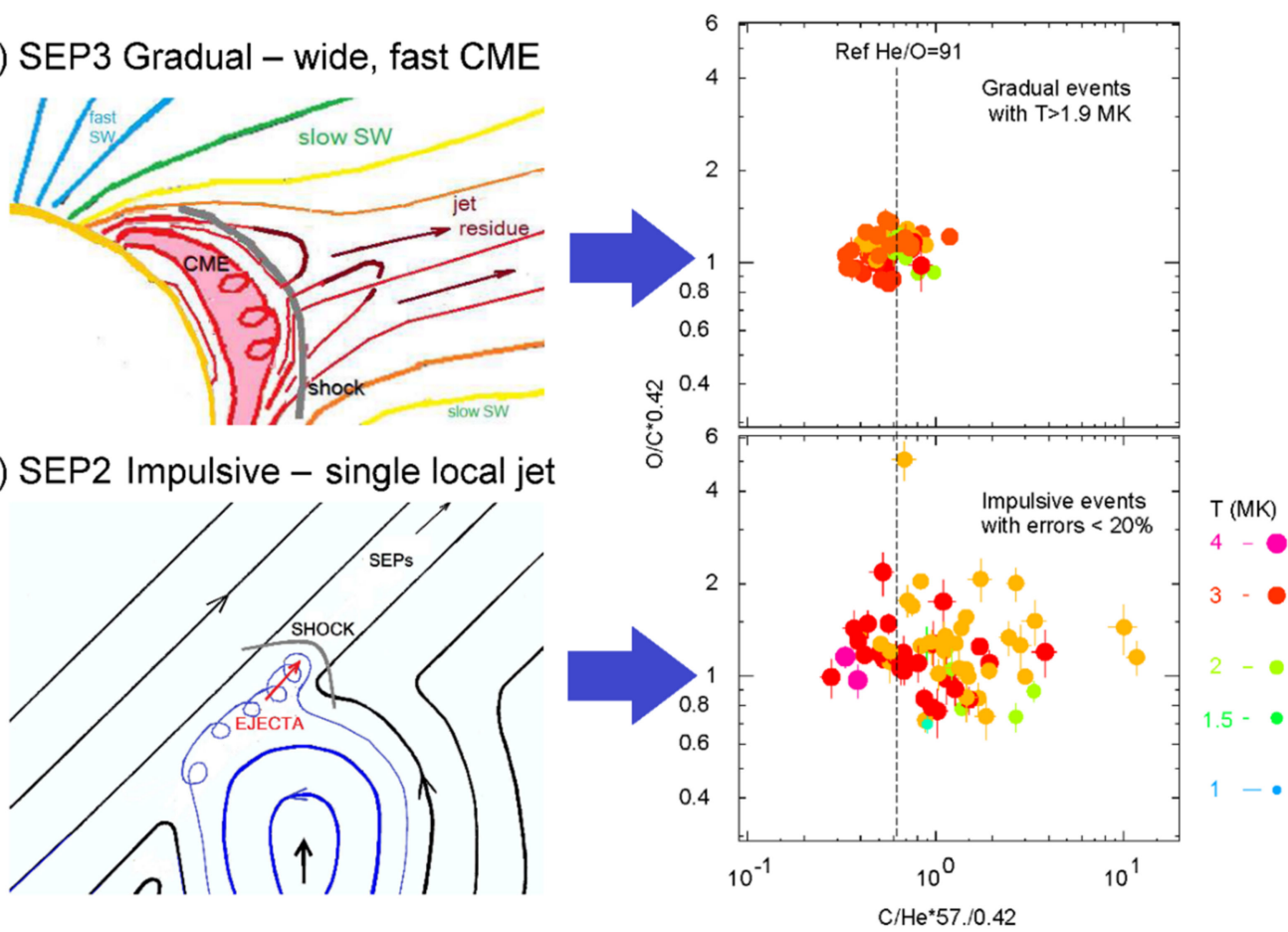

(a) SEP2 Impulsive - single local jet

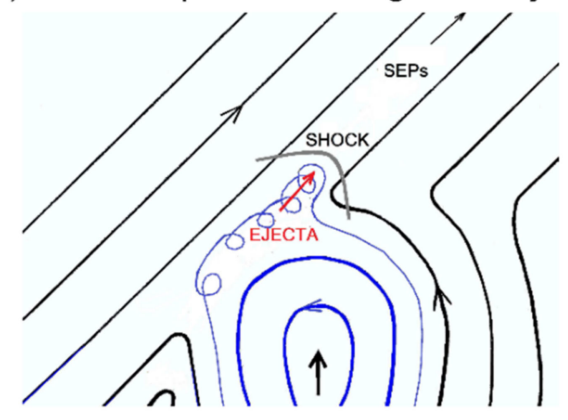

-

Figure 6. (a) Cartoon on the left shows SEP2 acceleration from magnetic reconnection in one of the individual jets leading to non-statistical spread in local abundances in $\mathrm{O} / \mathrm{C}$ vs. $\mathrm{C} / \mathrm{He}$ at $\mathrm{T}>2 \mathrm{MK}$ for various events on the right. (b) Cartoon on the left shows a shock traversing a pool of multi-jet residue producing an SEP3 event with $T>2 \mathrm{MK}$ with average abundances of $\mathrm{O} / \mathrm{C}$ vs. $\mathrm{C} / \mathrm{He}$ in each event as shown on the right $(82,8)$. The spread in abundance is reduced by pre-event averaging in multi-jet pools of SEP1 seed particles.

For the SEP3 events, the power law of abundances is determined by the seed population and the energy spectral indices by the shock wave, so these parameters are unrelated. However, for the SEP4 events, the shock determines both the abundances and the spectra, and they are roughly related $[114,125]$. If the power law of abundance enhancements is $(A / Q)^{\mathrm{x}}$ and the ion spectrum is $E^{\mathrm{y}}$, then we find that $y=x / 2-2$. The derivation of this numerical relationship is not clear. However, it specifies how shocks select ions of different $A / Q$ from the coronal plasma and relates to the "injection problem" of shock acceleration [126].

Energy spectral breaks [111] are an important feature of large gradual SEP events that also depend strongly upon A/Q [59,60,127-129] and provide a different way to study abundances.

\section{Conclusions and Questions}

The study of particle abundance in SEP events began sixty years ago and has expanded to a complete field of study in its own right. Progress in this field must speak for itself. Perhaps the most significant conclusions relate to the nature of the sources. We now understand the importance of CME-driven shock waves for acceleration in the large SEP events, and we have recently begun to associate the impulsive SEP events with magnetic reconnection on open field lines in solar jets. Solar flares also involve magnetic reconnection, but closed field lines prevent them from making any significant contribution to the SEPs we see in space. We are beginning to untangle the complexity of the seed population in shock acceleration, and we hope that added measurements by new spacecraft nearer to the Sun will better distinguish the effects of acceleration and transport.

Along with the progress, however, new questions have arisen:

1. Some variations in the abundance of $\mathrm{He}$ are not understood. SEP3 events have an average source $\mathrm{He} / \mathrm{O} \approx 91$ (e.g., Figure 6), while SEP4 events tend to have $\mathrm{He} / \mathrm{O} \approx 55$ [130]. This is usually attributed to the uniquely high FIP of $\mathrm{He}(24.6 \mathrm{eV})$, 
which can make He slow to ionize and enter the corona. Perhaps, but where is this low-He plasma located that is sampled for SEP4 events?

2. About $\sim 6 \%$ of impulsive SEP events are He-poor, where ${ }^{4} \mathrm{He} / \mathrm{O}$ is suppressed a factor of $\sim 10$ from the power-law fit line, even when $\mathrm{H}$ lies on that fit line [131]. Why?

3. The overall $\mathrm{C}$ abundance, i.e., $\mathrm{C} / \mathrm{O}=0.420 \pm 0.010$, in $\mathrm{SEPs}$ is uniquely far below the theoretical value, as shown in Figure 1, and far below the latest photospheric value of $\mathrm{C} / \mathrm{O}=0.589 \pm 0.054$ [14]. Event-to-event variations are small. The photospheric value was $\mathrm{C} / \mathrm{O}=0.479 \pm 0.055$ in 1996 [132], but has been rising ever since, while the SEPs have changed little. What is wrong with carbon? Is $\mathrm{C} / \mathrm{O}$ suppressed in SEPs (see Figure 1) or is the photospheric C/O too high? The FIP of $\mathrm{C}$ is not extreme, as with He.

4. Impulsive SEPs show enhancements of ${ }^{3} \mathrm{He}$ and of heavy elements, each large and each with its own mechanism. How and where do these mechanisms fit together in a jet, and how do they merge with jet models that do not yet consider SEP acceleration at all?

5. What is the origin of the relationship between power law element abundances and energy spectral indices in SEP4 shock events? Present SEP models treat source abundances as adjustable parameters, unrelated to the SEP reference abundances.

Funding: No institutional funding was provided for this work.

Conflicts of Interest: The author declares that this research was conducted in the absence of any commercial or financial relationships that could be construed as a potential conflict of interest.

\section{References}

1. Forbush, S.E. Three unusual cosmic ray increases possibly due to charged particles from the Sun. Phys. Rev. 1946, 70, 771. [CrossRef]

2. Kahler, S.W.; Sheeley, N.R., Jr.; Howard, R.A.; Koomen, M.J.; Michels, D.J.; McGuire, R.E.; von Rosenvinge, T.T.; Reames, D.V. Associations between coronal mass ejections and solar energetic proton events. J. Geophys. Res. 1984, 89, 9683. [CrossRef]

3. Fichtel, C.E.; Guss, D.E. Heavy nuclei in solar cosmic rays. Phys. Rev. Lett. 1961, 6, 495. [CrossRef]

4. Meyer, J.P. The baseline composition of solar energetic particles. Astrophys. J. 1985, 57, 151. [CrossRef]

5. Breneman, H.H.; Stone, E.C. Solar coronal and photospheric abundances from solar energetic particle measurements. Astrophys. J. Lett. 1985, 299, L57. [CrossRef]

6. Reames, D.V. Coronal Abundances determined from energetic particles. Adv. Space Res. 1995, 15, 41. [CrossRef]

7. Reames, D.V. Element abundances in solar energetic particles and the solar corona. Solar Phys. 2014, 289, 977. [CrossRef]

8. Reames, D.V. Solar Energetic Particles, 2nd ed.; Open access; Springer Nature: Cham, Switzerland, 2021. [CrossRef]

9. Laming, J.M. The FIP and inverse FIP effects in solar and stellar coronae. Living Rev. Sol. Phys. 2015, 12, 2. [CrossRef]

10. Mewaldt, R.A.; Cohen, C.M.S.; Leske, R.A.; Christian, E.R.; Cummings, A.C.; Stone, E.C.; von Rosenvinge, T.T.; Wiedenbeck, M.E. Fractionation of solar energetic particles and solar wind according to first ionization potential. Advan. Space Res. 2002, 30, 79. [CrossRef]

11. Reames, D.V. The "FIP effect" and the origins of solar energetic particles and of the solar wind. Solar Phys. 2018, 293, 47. [CrossRef]

12. Laming, J.M.; Vourlidas, A.; Korendyke, C. Element abundances: A new diagnostic for the solar wind. Astrophys. J. 2019, 879, 124. [CrossRef]

13. Caffau, E.; Ludwig, H.-G.; Steffen, M.; Freytag, B.; Bonofacio, P. Solar chemical abundances determined with a CO5BOLD 3D model atmosphere. Solar Phys. 2011, 268, 255. [CrossRef]

14. Asplund, M.; Amarsi, A.M.; Grevesse, N. The chemical make-up of the Sun: A 2020 vision, Astron. arXiv 2021, arXiv:2105.01661. (in press)

15. Wild, J.P.; Smerd, S.F.; Weiss, A.A. Solar Bursts. Annu. Rev. Astron. Astrophys. 1963, 1, 291. [CrossRef]

16. Lin, R.P. The emission and propagation of $40 \mathrm{keV}$ solar flare electrons. I: The relationship of $40 \mathrm{keV}$ electron to energetic proton and relativistic electron emission by the sun. Sol. Phys. 1970, 12, 266. [CrossRef]

17. Hsieh, K.C.; Simpson, J.A. Galactic ${ }^{3} \mathrm{He}$ above $10 \mathrm{MeV}$ per nucleon and the solar contributions of hydrogen and helium. Astrophys. J. Lett. 1970, 162, L197. [CrossRef]

18. Serlemitsos, A.T.; Balasubrahmanyan, V.K. Solar particle events with anomalously large relative abundance of ${ }^{3} \mathrm{He}$. Astrophys. J. 1975, 198, 195. [CrossRef]

19. McGuire, R.E.; von Rosenvinge, T.T.; McDonald, F.B. A survey of solar cosmic ray composition. Int. Cosm. Ray Conf. Tokyo 1979, 5, 61.

20. Cook, W.R.; Stone, E.C.; Vogt, R.E. Elemental composition of solar energetic particles. Astrophys. J. 1984, 279, 827. [CrossRef]

21. Mason, G.M.; Reames, D.V.; Klecker, B.; Hovestadt, D.; von Rosenvinge, T.T. The heavy-ion compositional signature in He-3-rich solar particle events. Astrophys. J. 1986, 303, 849. [CrossRef] 
22. Reames, D.V.; Meyer, J.P.; von Rosenvinge, T.T. Energetic-particle abundances in impulsive solar flare events. Astrophys. J. Suppl. 1994, 90, 649. [CrossRef]

23. Reames, D.V. Abundances of trans-iron elements in solar energetic particle events. Astrophys. J. Lett. 2000, 540, L111. [CrossRef]

24. Reames, D.V.; Ng, C.K. Heavy-element abundances in solar energetic particle events. Astrophys. J. 2004, 610, 510. [CrossRef]

25. Mason, G.M.; Mazur, J.E.; Dwyer, J.R.; Jokippi, J.R.; Gold, R.E.; Krimigis, S.M. Abundances of heavy and ultraheavy ions in ${ }^{3}$ He-rich solar flares. Astrophys. J. 2004, 606, 555. [CrossRef]

26. Reames, D.V.; Cliver, E.W.; Kahler, S.W. Abundance enhancements in impulsive solar energetic-particle events with associated coronal mass ejections. Solar Phys. 2014, 289, 3817. [CrossRef]

27. Reames, D.V. Particle acceleration at the Sun and in the heliosphere. Space Sci. Rev. 1999, 90, 413. [CrossRef]

28. Ho, G.C.; Roelof, E.C.; Mason, G.M. The upper limit on ${ }^{3}$ He fluence in solar energetic particle events. Atrophys. J. Lett. 2005, 621, L141. [CrossRef]

29. Mason, G.M. ${ }^{3}$ He-rich solar energetic particle events. Space Sci. Rev. 2007, 130, 231. [CrossRef]

30. Reames, D.V.; von Rosenvinge, T.T.; Lin, R.P. Solar ${ }^{3}$ He-rich events and nonrelativistic electron events-A new association. Astrophys. J. 1985, 292, 716. [CrossRef]

31. Reames, D.V.; Stone, R.G. The identification of solar ${ }^{3}$ He-rich events and the study of particle acceleration at the sun. Astrophys. J. 1986, 308, 902. [CrossRef]

32. Ibragimov, I.A.; Kocharov, G.E. Possible mechanism for enrichment of solar cosmic rays by helium-three and heavy nuclei. Int Conf. Cosm. Rays 1977, 11, 340.

33. Kocharov, G.E.; Kocharov, L.G. Present state of experimental and theoretical investigations of solar events enriched by helium-3. Cosmophysics 1978, 37, 37-72.

34. Kocharov, G.E.; Kocharov, L.G. ${ }^{3}$ He-rich solar flares. Space Science Rev. 1984, 38, 89. [CrossRef]

35. Weatherall, J. Turbulent heating in solar cosmic-ray theory. Astrophys. J. 1984, 281, 468. [CrossRef]

36. Fisk, L.A. ${ }^{3}$ He-rich flares-A possible explanation. Astrophys. J. 1978, 224, 1048. [CrossRef]

37. Varvoglis, H.; Papadopoulis, K. Selective nonresonant acceleration of $\mathrm{He}-3(2+)$ and heavy ions by $\mathrm{H}(+)$ cyclotron waves. Astrophys. J. Lett. 1983, 270, L95. [CrossRef]

38. Winglee, R.M. Heating and acceleration of heavy ions during solar flares. Astrophys. J. 1989, 343, 511. [CrossRef]

39. Riyopoulos, S. Subthreshold stochastic diffusion with application to selective acceleration of ${ }^{3}$ He in solar flares. Astrophys. J. 1991, 381, 578. [CrossRef]

40. Temerin, M.; Roth, I. The production of ${ }^{3} \mathrm{He}$ and heavy ion enrichment in ${ }^{3} \mathrm{He}-\mathrm{rich}$ flares by electromagnetic hydrogen cyclotron waves. Astrophys. J. Lett. 1992, 391, L105. [CrossRef]

41. Drake, J.F.; Cassak, P.A.; Shay, M.A.; Swisdak, M.; Quataert, E. A magnetic reconnection mechanism for ion acceleration and abundance enhancements in impulsive flares. Astrophys. J. Lett. 2009, 700, L16. [CrossRef]

42. Arnold, H.; Drake, J.; Swisdak, M.; Guo, F.; Dahlin, J.; Chen, B.; Fleishman, G.; Glesener, L.; Kontar, E.; Phan, T.; et al. Electron acceleration during macroscale magnetic reconnection. arXiv 2011, arXiv:2011.01147.

43. Luhn, A.; Klecker, B.; Hovestadt, D.; Gloeckler, G.; Ipavich, F.M.; Scholer, M.; Fan, C.Y.; Fisk, L.A. Ionic charge states of N, Ne, Mg, $\mathrm{Si}$ and $\mathrm{S}$ in solar energetic particle events. Adv. Space Res. 1984, 4, 161. [CrossRef]

44. Luhn, A.; Klecker, B.; Hovestadt, D.; Möbius, E. The mean ionic charge of silicon in He-3-rich solar flares. Astrophys. J. 1987, 317, 951. [CrossRef]

45. DiFabio, R.; Guo, Z.; Möbius, E.; Klecker, B.; Kucharek, H.; Mason, G.M.; Popecki, M. Energy-dependent charge states and their connection with ion abundances in impulsive solar energetic particle events. Astrophys. J. 2008, 687, 623. [CrossRef]

46. Newkirk, G., Jr.; Wenzel, D.G. Rigidity-independent propagation of cosmic rays in the solar corona. J. Geophys. Res. 1978, 83, 2009. [CrossRef]

47. Mason, G.M.; Gloeckler, G.; Hovestadt, D. Temporal variations of nucleonic abundances in solar flare energetic particle events. II-Evidence for large-scale shock acceleration. Astrophys. J. 1984, 280, 902. [CrossRef]

48. Reames, D.V. Bimodal abundances in the energetic particles of solar and interplanetary origin. Astrophys. J. Lett. 1988, 330 , L71. [CrossRef]

49. Reames, D.V. Magnetic topology of impulsive and gradual solar energetic particle events. Astrophys. J. Lett. 2002, 571 , L63. [CrossRef]

50. Gosling, J.T. The solar flare myth. J. Geophys. Res. 1993, 98, 18937. [CrossRef]

51. Gosling, J.T. Corrections to "The solar flare myth". J. Geophys. Res. 1994, 99, 4259. [CrossRef]

52. Zirin, H. Solar storminess, Sky and Telescope. 9 November 1994.

53. Hudson, H.S. Solar flares: No "myth". Eos Trans. 1995, AGU 76, 405. [CrossRef]

54. Miller, J.A. Much ado about nothing. Eos Trans. 1995, AGU 76, 401. [CrossRef]

55. Reames, D.V. The dark side of the solar flare myth. Eos Trans. 1995, AGU 76, 401. [CrossRef]

56. Reames, D.V. Solar energetic particles: A paradigm shift. Revs. Geophys. Suppl. 1995, 33, 585. [CrossRef]

57. Mason, G.M.; Mazur, J.E.; Dwyer, J.R. 3He enhancements in large solar energetic particle events. Astrophys. J. Lett. 1999, 525, L133. [CrossRef] [PubMed] 
58. Tylka, A.J.; Cohen, C.M.S.; Dietrich, W.F.; Maclennan, C.G.; McGuire, R.E.; Ng, C.K.; Reames, D.V. Evidence for remnant flare suprathermals in the source population of solar energetic particles in the 2000 bastille day event. Astrophys. J. Lett. 2001, 558, L59. [CrossRef]

59. Tylka, A.J.; Cohen, C.M.S.; Dietrich, W.F.; Lee, M.A.; Maclennan, C.G.; Mewaldt, R.A.; Ng, C.K.; Reames, D.V. Shock geometry, seed populations, and the origin of variable elemental composition at high energies in large gradual solar particle events. Astrophys. J. 2005, 625, 474. [CrossRef]

60. Tylka, A.J.; Lee, M.A. Spectral and compositional characteristics of gradual and impulsive solar energetic particle events. Astrophys. J. 2006, 646, 1319. [CrossRef]

61. Richardson, I.G.; Reames, D.V.; Wenzel, K.P.; Rodriguez-Pacheco, J. Quiet-time properties of $<10 \mathrm{MeV} / \mathrm{n}$ interplanetary ions during solar maximum and minimum. Astrophys. J. Lett. 1990, 363, L9. [CrossRef]

62. Desai, M.I.; Mason, G.M.; Dwyer, J.R.; Mazur, J.E.; Gold, R.E.; Krimigis, S.M.; Smith, C.W.; Skoug, R.M. Evidence for a suprathermal seed population of heavy ions accelerated by interplanetary shocks near 1 AU. Astrophys. J. 2003, 588, 1149. [CrossRef]

63. Wiedenbeck, M.E.; Cohen, C.M.S.; Cummings, A.C.; de Nolfo, G.A.; Leske, R.A.; Mewaldt, R.A.; Stone, E.C.; von Rosenvinge, T.T. Persistent energetic 3He in the inner heliosphere. Int. Cosm. Ray Conf. 2008, 1, 91.

64. Bučík, R.; Innes, D.E.; Mall, U.; Korth, A.; Mason, G.M.; Gómez-Herrero, R. Multi-spacecraft observations of recurrent 3He-rich solar energetic particles. Astrophys. J. 2014, 786, 71. [CrossRef]

65. Bučík, R.; Innes, D.E.; Chen, N.H.; Mason, G.M.; Gómez-Herrero, R.; Wiedenbeck, M.E. Long-lived energetic particle source regions on the Sun. J. Phys. Conf. Ser. 2015, 642, 012002. [CrossRef]

66. Chen, N.H.; Bučík, R.; Innes, D.E.; Mason, G.M. Case studies of multi-day 3He-rich solar energetic particle periods. Astron. Astrophys. 2015, 580, 16. [CrossRef]

67. Kahler, S.W.; Reames, D.V.; Sheeley, N.R., Jr. Coronal mass ejections associated with impulsive solar energetic particle events. Astrophys. J. 2001, 562, 558. [CrossRef]

68. Shimojo, M.; Shibata, K. Physical parameters of solar X-ray jets. Astrophys. J. 2000, 542, 1100. [CrossRef]

69. Ramaty, R.; Murphy, R.J. Nuclear processes and accelerated particles in solar flares. Space Sci. Rev. 1987, 45, 213. [CrossRef]

70. Mandzhavidze, N.; Ramaty, R.; Kozlovsky, B. Determination of the abundances of subcoronal $4 \mathrm{He}$ and of solar flare-accelerated $3 \mathrm{He}$ and $4 \mathrm{He}$ from gamma-ray spectroscopy. Astrophys. J. 1999, 518, 918. [CrossRef]

71. Murphy, R.J.; Kozlovsky, B.; Share, G.H. Evidence for enhanced 3He in flare-accelerated particles based on new calculations of the gamma-ray line spectrum. Astrophys. J. 2016, 833, 166. [CrossRef]

72. Murphy, R.J.; Ramaty, R.; Kozlovsky, B.; Reames, D.V. Solar abundances from gamma-ray spectroscopy: Comparisons with energetic particle, photospheric, and coronal abundances. Astrophys. J. 1991, 371, 793. [CrossRef]

73. Nitta, N.V.; Reames, D.V.; DeRosa, M.L.; Yashiro, S.; Gopalswamy, N. Solar sources of impulsive solar energetic particle events and their magnetic field connection to the earth. Astrophys. J. 2006, 650, 438. [CrossRef]

74. Wang, Y.-M.; Pick, M.; Mason, G.M. Coronal holes, jets, and the origin of 3He-rich particle events. Astrophys. J. 2006, 639, 495. [CrossRef]

75. Ko, Y.-K.; Tylka, A.J.; Ng, C.K.; Wang, Y.-M.; Dietrich, W.F. Source regions of the interplanetary magnetic field and variability in heavy-ion elemental composition in gradual solar energetic particle events. Astrophys. J. 2013, 776, 92. [CrossRef]

76. Bučík, R.; Innes, D.E.; Mason, G.M.; Wiedenbeck, M.E.; Gómez-Herrero, R.; Nitta, N.V. 3He-rich solar energetic particles in helical jets on the Sun. Astrophys. J. 2018, 852, 76. [CrossRef]

77. Bučík, R.; Wiedenbeck, M.E.; Mason, G.M.; Gómez-Herrero, R.; Nitta, N.V.; Wang, L. 3He-rich solar energetic particles from sunspot jets. Astrophys. J. Lett. 2018, 869, L21. [CrossRef]

78. Bučík, R.; Mulay, S.M.; Mason, G.M.; Nitta, N.V.; Desai, M.I.; Dayeh, M.A. Temperature in solar sources of 3He-rich solar energetic particles and relation to ion abundances. Astrophys. J. 2021, 908, 243. [CrossRef]

79. Bučík, R. 3He-rich solar energetic particles: Solar sources. Space Sci. Rev. 2020, 216, 24. [CrossRef]

80. Reames, D.V.; Cliver, E.W.; Kahler, S.W. Variations in abundance enhancements in impulsive solar energetic-particle events and related CMEs and flares. Solar Phys. 2014, 289, 4675. [CrossRef]

81. Reames, D.V. Abundances, ionization states, temperatures, and FIP in solar energetic particles. Space Sci. Rev. 2018, $214,61$. [CrossRef]

82. Reames, D.V. Four distinct pathways to the element abundances in solar energetic particles. Space Sci. Rev. 2020, 216, 20. [CrossRef]

83. Reames, D.V. Hydrogen and the abundances of elements in impulsive solar energetic-particle events. Solar Phys. 2019, $294,37$. [CrossRef]

84. Reames, D.V.; Cliver, E.W.; Kahler, S.W. Temperature of the source plasma for impulsive solar energetic particles. Sol. Phys. 2015, 290, 1761. [CrossRef]

85. Lee, M.A. Coupled hydromagnetic wave excitation and ion acceleration at interplanetary traveling shocks. J. Geophys. Res. 1983, 88,6109 . [CrossRef]

86. Lee, M.A. Coupled hydromagnetic wave excitation and ion acceleration at an evolving coronal/interplanetary shock. Astrophys. J. Suppl. 2005, 158, 38. [CrossRef]

87. Jones, F.C.; Ellison, D.E. The plasma physics of shock acceleration. Space Sci. Rev. 1991, 58, 259. [CrossRef] 
88. Zank, G.P.; Rice, W.K.M.; Wu, C.C. Particle acceleration and coronal mass ejection driven shocks: A theoretical model. J. Geophys. Res. 2000, 105, 25079. [CrossRef]

89. Zank, G.P.; Li, G.; Verkhoglyadova, O. Particle Acceleration at Interplanetary Shocks. Space Sci. Rev. 2007, 130, 255. [CrossRef]

90. Cliver, E.W.; Kahler, S.W.; Reames, D.V. Coronal shocks and solar energetic proton events. Astrophys. J. 2004, 605, 902. [CrossRef]

91. Sandroos, A.; Vainio, R. Simulation results for heavy ion spectral variability in large gradual solar energetic particle events. Astrophys. J. 2007, 662, L127. [CrossRef]

92. Ng, C.K.; Reames, D.V. Shock acceleration of solar energetic protons: The first 10 minutes. Astrophys. J. Lett. 2008, 686, L123. [CrossRef]

93. Reames, D.V. The two sources of solar energetic particles. Space Sci. Rev. 2013, 175, 53. [CrossRef]

94. Lee, M.A.; Mewaldt, R.A.; Giacalone, J. Shock acceleration of ions in the heliosphere. Space Sci. Rev. 2012, 173, 247. [CrossRef]

95. Desai, M.I.; Giacalone, J. Large gradual solar energetic particle events. Living Rev. Sol. Phys. 2016, 13, 1-32. [CrossRef]

96. Reames, D.V.; Barbier, L.M.; Ng, C.K. The spatial distribution of particles accelerated by coronal mass ejection-driven shocks. Astrophys. J. 1996, 466, 473. [CrossRef]

97. Reames, D.V.; Kahler, S.W.; Ng, C.K. Spatial and temporal invariance in the spectra of energetic particles in gradual solar events. Astrophys. J. 1997, 491, 414. [CrossRef]

98. Rouillard, A.C.; Odstrčil, D.; Sheeley, N.R., Jr.; Tylka, A.J.; Vourlidas, A.; Mason, G.; Wu, C.-C.; Savani, N.P.; Wood, B.E.; Ng, C.K.; et al. Interpreting the properties of solar energetic particle events by using combined imaging and modeling of interplanetary shocks. Astrophys. J. 2011, 735, 7. [CrossRef]

99. Rouillard, A.; Sheeley, N.R., Jr.; Tylka, A.; Vourlidas, A.; Ng, C.K.; Rakowski, C.; Cohen, C.M.S.; Mewaldt, R.A.; Mason, G.M.; Reames, D.; et al. The longitudinal properties of a solar energetic particle event investigated using modern solar imaging. Astrophys. J. 2012, 752, 44. [CrossRef]

100. Rouillard, A.P.; Plotnikov, I.; Pinto, R.F.; Tirole, M.; Lavarra, M.; Zucca, P.; Vainio, R.; Tylka, A.J.; Vourlidas, A.; De Rosa, M.L.; et al. Deriving the properties of coronal pressure fronts in 3D: Application to the 2012 May 17 ground level enhancement. Astrophys. J. 2016, 833, 45. [CrossRef]

101. Kahler, S.W. The correlation between solar energetic particle peak intensities and speeds of coronal mass ejections: Effects of ambient particle intensities and energy spectra. J. Geophys. Res. 2001, 106, 20947. [CrossRef]

102. Kouloumvakos, A.; Rouillard, A.P.; Wu, Y.; Vainio, R.; Vourlidas, A.; Plotnikov, I.; Afanasiev, A.; Önel, H. Connecting the properties of coronal shock waves with those of solar energetic particles. Astrophys. J. 2019, 876, 80. [CrossRef]

103. Kahler, S.W. Injection profiles of solar energetic particles as functions of coronal mass ejection heights. Astrophys. J. 1994, $428,837$. [CrossRef]

104. Tylka, A.J.; Cohen, C.M.S.; Dietrich, W.F.; Krucker, S.; McGuire, R.E.; Mewaldt, R.A.; Ng, C.K.; Reames, D.V.; Share, G.H. Contributed papers v. 6 (SH sessions 1.1-2.3). In Proceedings of the 28th International Cosmic Ray Conference (Tsukuba) 63305 (2003), Tsukuba, Japan, 31 July-7 August 2003.

105. Reames, D.V. Solar release times of energetic particles in ground-level events. Astrophys. J. 2009, 693, 812. [CrossRef]

106. Reames, D.V. Solar energetic-particle release times in historic ground-level events. Astrophys. J. 2009, 706, 844. [CrossRef]

107. Cliver, E.W.; Ling, A.G. Electrons and protons in solar energetic particle events. Astrophys. J. 2007, 658, 1349. [CrossRef]

108. Cliver, E.W. Flare vs. shock acceleration of high-energy protons in solar energetic particle events. Astrophys. J. 2016, 832, 128. [CrossRef]

109. Tylka, A.J.; Dietrich, W.F. A New and Comprehensive Analysis of Proton Spectra in Ground-Level Enhanced (GLE) Solar Particle Events. In Proceedings of the 31st International Cos. Ray Conference Lódz, Lódz, Poland, 8 July 2009; Available online: http:/ / icrc2009.uni.lodz.pl/proc/pdf/icrc0273.pdf (accessed on 7 August 2021).

110. Gopalswamy, N.; Xie, H.; Yashiro, S.; Akiyama, S.; Mäkelä, P.; Usoskin, I.G. Properties of Ground level enhancement events and the associated solar eruptions during solar cycle 23. Space Sci. Rev. 2012, 171, 23. [CrossRef]

111. Mewaldt, R.A.; Looper, M.D.; Cohen, C.M.S.; Haggerty, D.K.; Labrador, A.W.; Leske, R.A.; Mason, G.M.; Mazur, J.E.; von Rosenvinge, T.T. Energy spectra, composition, other properties of ground-level events during solar cycle 23. Space Sci. Rev. 2012, 171, 97. [CrossRef]

112. Reames, D.V. Hydrogen and the abundances of elements in gradual solar energetic-particle events. Solar Phys. 2019, $294,69$. [CrossRef]

113. Reames, D.V. Temperature of the source plasma in gradual solar energetic particle events. Solar Phys. 2016, 291, 911. [CrossRef]

114. Reames, D.V. Distinguishing the rigidity dependences of acceleration and transport in solar energetic particles. Solar Phys. 2020, 295, 113. [CrossRef]

115. Mason, G.M.; Ng, C.K.; Klecker, B.; Green, G. Impulsive acceleration and scatter-free transport of about $1 \mathrm{MeV}$ per nucleon ions in 3He-rich solar particle events. Astrophys. J. 1989, 339, 529. [CrossRef]

116. Stix, T.H. Waves in Plasmas; AIP: New York, NY, USA, 1992.

117. Reames, D.V. Acceleration of energetic particles by shock waves from large solar flares. Astrophys. J. Lett. 1990,358, L63. [CrossRef]

118. Reames, D.V.; Ng, C.K.; Tylka, A.J. Initial time dependence of abundances in solar particle events. Astrophys. J. Lett. 2000, 531 , L83. [CrossRef] [PubMed] 
119. Ng, C.K.; Reames, D.V. Focused interplanetary transport of approximately $1 \mathrm{MeV}$ solar energetic protons through self-generated Alfven waves. Astrophys. J. 1994, 424, 1032. [CrossRef]

120. Ng, C.K.; Reames, D.V.; Tylka, A.J. Effect of proton-amplified waves on the evolution of solar energetic particle composition in gradual events. Geophys. Res. Lett. 1999, 26, 2145. [CrossRef]

121. Ng, C.K.; Reames, D.V.; Tylka, A.J. Modeling shock-accelerated solar energetic particles coupled to interplanetary Alfvén waves. Astrophys. J. 2003, 591, 461. [CrossRef]

122. Ng, C.K.; Reames, D.V.; Tylka, A.J. Solar energetic particles: Shock acceleration and transport through self-amplified waves. AIP Conf. Proc. 2012, 1436, 212. [CrossRef]

123. Reames, D.V.; Ng, C.K. Streaming-limited intensities of solar energetic particles. Astrophys. J. 1998, 504, 1002. [CrossRef]

124. Reames, D.V.; $\mathrm{Ng}$, C.K. Streaming-limited intensities of solar energetic particles on the intensity plateau. Astrophys. J. 2010, 723, 1286. [CrossRef]

125. Reames, D.V. The correlation between energy spectra and element abundances in solar energetic particles. Sol. Phys. 2021, 296, 24. [CrossRef]

126. Zank, G.P.; Rice, W.K.M.; le Roux, J.A.; Cairns, I.H.; Webb, G.M. The “injection problem" for quasiparallel shocks. Phys. Plasmas 2001, 8, 4560. [CrossRef]

127. Li, G.; Zank, G.P.; Rice, W.K.M. Acceleration and transport of heavy ions at coronal mass ejection-driven shocks. J. Geophys. Res. 2005, 110, A06104. [CrossRef]

128. Li, G.; Zank, G.P.; Verkhoglyadova, O.; Mewaldt, R.A.; Cohen, C.M.S.; Mason, G.M.; Desai, M.I. Shock geometry and spectral breaks in large SEP events. Astrophys. J. 2009, 702, 998. [CrossRef]

129. Zhao, L.; Zhang, M.; Rassoul, H. Double power laws in the event-integrated solar energetic particle spectrum. Astrophys. J. 2016, 821, 62. [CrossRef]

130. Reames, D.V. The abundance of helium in the source plasma of solar energetic particles. Solar Phys. 2017, 292, 156. [CrossRef]

131. Reames, D.V. Helium suppression in impulsive solar energetic-particle events. Sol. Phys. 2019, 294, 32. [CrossRef]

132. Grevesse, N.; Noels, A.; Sauval, A.J. Standard Abundances. Astron. Soc. Pacif. Conf. Ser. 1996, 99, 117. 